Código JEL: L83, M31

\title{
Estudio del perfil de la demanda del turismo ornitológico en el Prepirineo oscense
}

\author{
Laura SANTOLARIA MÁS \\ laurasantolaria86@gmail.com \\ Helena GINÉ ABAD (dir.) \\ hgine@unizar.es \\ Máster en Planificación y Gestión del turismo \\ Facultad de Empresa y Gestión Pública de Huesca (Universidad de Zaragoza)
}

Recibido: 10-01-2013

Aceptado: 21-01-2013

\section{RESUMEN}

El ornitoturismo está considerado como una actividad sostenible y propulsora tanto económica como socialmente del territorio.

Esta tipología de turismo, se ha ido desarrollando con el paso del tiempo, pero no se da como propia actividad de ocio hasta el S.XVIII. Comenzó en Inglaterra, pero en España no ha surgido hasta hace dos décadas. En el Prepirineo oscense, cada vez está ganando más adeptos canalizando flujos desde Europa e incluso América.

El objetivo principal de este estudio es conocer el perfil de la demanda interesada en el avistamiento de la avifauna del lugar. Para ello se ha investigado el sector, las figuras de protección del espacio y demás aspectos vinculados al turismo ornitológico. Se han realizado entrevistas a instituciones, para conocer sus proyectos en el territorio, y al sector hotelero y empresarial. Igualmente se diseñó una encuesta dirigida a los turistas ornitológicos para conocer sus perfiles y preferencias.

Palabras clave: turismo ornitológico, perfil de la demanda, prepirineo oscense. 


\title{
Profile study of birdwatching tourism demand in the Pyrenees
}

\begin{abstract}
Birding is considered as a sustainable activity and driving economically and socially the territory.

This kind of tourism has been developed along the time, but it wasn't known as leisure activity until the eighteenth century. It began in England. In Spain, has emerged two decades ago. The Pyrenees, gain more and more followers, especially tourists coming from Europe and even America.

The main objective of these studies is to know the demand profile interested in birdwatching. For that, this sector has been investigated, also the protection figures in the area and other aspects related to birdwatchers. There have been interviews with institutions, to know their projects in the territory and the hospitality and corporate sector. Also a survey was designed to know tourists profiles and preferences.
\end{abstract}

Keywords: Birdwatching, birding, demand profile, Pyrenees

Sumario: 1. Introducción. 2. Normativa internacional y nacional de protección de aves. 3 . Programas a nivel nacional para el fomento del turismo ornitológico. 4. Turismo ornitológico en Aragón. 5. Turismo ornitológico en las comarcas de estudio (Hoya de Huesca y Somontano de Barbastro). 6. Análisis y resultados. 7. Conclusiones e implicaciones.

\section{INTRODUCCIÓN}

La ornitología ha estado presente en el ser humano desde el principio de los siglos. Comenzó como simples referencias a la caza y supervivencia del hombre. Con el tiempo se fue convirtiendo en objeto de estudio por sus migraciones y surgieron escritos sobre éstas, lo que supuso un gran avance para la ciencia. Pero fue realmente en el S.XVIII y mediados del S.XIX cuando se concibe como una actividad de ocio (Moss, S. 2004 y De Farias 2007).

El origen del ornitoturismo se encuentra en Inglaterra, al fundarse en 1889 "The Royal Society for the Protection of Birds" (R.S.P.B.), de la cual han partido las diferentes actuaciones para la protección y difusión ligadas a esta actividad (Fernández Tabales, A., Beltrán de Ceballos, Carmona Arteaga, JM. y Mainar Aguilar, M. 2007).

El turismo ornitológico, se puede definir como una modalidad de turismo que se localiza en el medio natural y tiene como objetivo principal el avistamiento e identificación de las aves del lugar, el conocimiento del entorno que las rodea y el hábitat en el cual se mueven (Blanco Portillo, R. 2012). Es respetuoso con el medio ambiente y suele centrarse en zonas rurales, lo que puede significar una aportación socio-económica extra importante para la población. Además es 
desestacionalizador, ya que los turistas realizan sus viajes en función de la avifauna que desean observar (De Juan, J. 2006, López Roig, J. 2008).

Este trabajo pretende profundizar en el conocimiento de este sector. El proceso seguido en la elaboración ha sido el siguiente. En primer lugar se ha realizado una revisión bibliográfica en la que destacan los antecedentes y la evolución histórica, basada especialmente en los estudios de las principales asociaciones.

A continuación, se revisa la normativa de protección de las aves, tanto nacional como internacional, en las que se encuentran figuras tan importantes como la Directiva de Aves Silvestres, la Directiva de Hábitats, la Red Natura 2000 la cual está integrada por Zonas de Especial Protección de Aves (ZEPA), Lugares de Interés comunitario (LIC) y Zonas Especiales de Conservación (ZEC).

Al igual que los diferentes programas que se han realizado hasta el momento, como serían el Proyecto LIFE, TRINO, RETO ó IBERAVES.

Posteriormente se realizó un estudio de este tipo de turismo en Aragón, desarrollando la normativa regional, los proyectos que se han llevado a cabo, las rutas más emblemáticas, así como las asociaciones más importantes.

Finaliza el estudio descendiendo al ámbito territorial, es decir, la comarca de la Hoya de Huesca y del Somontano de Barbastro, en las que se sigue el mismo esquema, una descripción del territorio, delimitación comarcal, actuaciones de fomento llevadas a cabo, centros de información y avistamiento de aves que existen y rutas más importantes.

Se llevó a cabo la encuesta durante los meses de febrero a mayo del 2013, a todos aquellos turistas que se encontraban en el área de estudio, cuya principal motivación era la avistación y el reconocimiento de la avifauna del territorio.

\section{NORMATIVA INTERNACIONAL Y NACIONAL DE PROTECCIÓN DE AVES}

Existen diversas figuras de protección de las aves a nivel europeo y nacional las cuales tienen una gran importancia para la conservación de los ecosistemas en base a las aves y su sistema migratorio. Entre estas destacamos varias directivas:

Directiva de Aves Silvestres: fue aprobada por la Unión Europea en el año 1979 y tuvo como objetivo la protección, administración y regulación de las diferentes especies de aves que viven en estado silvestre, por lo que obligó a todos los Estados miembros a tomar ciertas medidas necesarias para mantener las poblaciones de las diferentes especies de aves.

Directiva Hábitats: Surge en el Convenio sobre la Biodiversidad, firmado por más de un centenar de países en la Cumbre de la Tierra de Río de Janeiro de 1992. $\mathrm{Su}$ objetivo es garantizar la biodiversidad por medio de la conservación de los hábitats naturales y de la fauna y flora silvestres en el territorio europeo y para ello 
se instaura un régimen de protección de los hábitats y un régimen de protección directa de las especies contra la caza, comercialización, etc.

Red Natura 2000: La Red Natura 2000 es "Una red ecológica europea coherente de zonas especiales de conservación" "I. Dicha red, tiene que garantizar el mantenimiento o el restablecimiento, de un estado de conservación favorable, de los tipos de hábitats naturales y de los hábitats de las especies de que se trate en su área de distribución natural.

Esta Red está integrada por:

- Zonas de Especial Protección para las Aves (ZEPA). Estas son lugares que cada estado miembro de la Unión Europea debe designar para proteger una serie de aves. Pueden ser zonas tanto terrestres como marinas, en las que se intenta que las aves amenazadas cuenten con lugares seguros y con buena calidad del hábitat en el que viven y se reproducen para garantizar su conservación.

- Lugar de Interés Comunitario (LIC). Lugares que en las zonas en las que se declara, contribuyen a mantener y restablecer el tipo de hábitat natural o una especie, dentro de un estado de conservación favorable y que pueda contribuir de forma coherente con la Red Natura 2000 y que por lo tanto mantenga la diversidad biológica de la región de que se trate.

- Zonas Especiales de Conservación (ZEC), definidos como "un lugar de importancia comunitaria designado por los Estados miembros mediante un acto reglamentario, administrativo y/o contractual, en el cual se aplican las medidas de conservación necesarias para el mantenimiento o el restablecimiento, en un estado de conservación favorable, de los hábitats naturales y/o de las poblaciones de las especies para las cuales se haya designado el lugar ${ }^{2}$.

Además de las directivas anteriormente citadas, que afectan al territorio español, existe una normativa para la protección del medio natural a nivel nacional de 1989 y se encuentra recopilada de la "Ley de Conservación de los Espacios Naturales y la Flora y la Fauna Silvestre"3. Dicha Ley establece cuatro figuras para la protección de los Espacios Naturales:

- Parques Naturales: son áreas naturales, poco transformadas por la actividad del ser humano, que debido a la belleza de sus paisajes, la importancia de

\footnotetext{
${ }^{1}$ www.rednatura.es

${ }^{2}$ www.rednatura.es

${ }^{3}$ https://www.boe.es/buscar/doc.php?id=BOE-A-1989-6881
} 
sus ecosistemas o la singularidad de la flora, la fauna o formaciones geomorfológicas, poseen unos ciertos valores cuya conservación merece una atención predominante.

- Reservas Naturales: son espacios naturales cuya finalidad es la protección de ecosistemas, comunidades o elementos biológicos, que por su rareza, fragilidad, importancia o singularidad tienen que tener una valoración especial. Dentro de dicha área está limitada la explotación de los recursos, así como la recolección de materiales geológicos o biológicos.

- Monumentos Naturales: son espacios en la naturaleza constituidos por formaciones singulares, y que por lo tanto tienen que ser protegidos y conservados de forma especial.

- Paisajes Protegidos: son lugares concretos del medio natural que por ciertos valores estéticos y culturales, necesitan una protección especial.

Esta Ley además define unas Zonas Periféricas de Protección, para amortiguar los posibles impactos que puedan surgir de las actividades desarrolladas en los alrededores de cada Espacio Protegido.

\section{PROGRAMAS A NIVEL NACIONAL PARA EL FOMENTO DEL TURISMO ORNITOLÓGICO Proyecto TRINO ${ }^{4}$ (Turismo Rural de Interior y Ornitología)}

Es una propuesta de treinta Grupos de Acción Local (GAL) del programa LEADER y PRODERCAL del Eje 4 del Fondo Europeo Agrícola de Desarrollo Rural (FEADER) en Castilla y León.

Las comarcas en las que actúan dichos GAL cuentan con más de $500 \mathrm{~km}$ de rutas señalizadas, observatorios y otras infraestructuras para la observación de avifauna. Este ámbito geográfico posee un gran potencial para la observación de aves puesto que cuenta con una amplia variedad de ecosistemas que le proporcionan una alta diversidad avifaunística.

Los objetivos ${ }^{5}$ más destacables que pretende llevar a cabo TRINO son:

\footnotetext{
${ }^{4} \mathrm{http}: / / \mathrm{www} \cdot$ birdwatchinginspain.com/index.php/es/actividades

${ }^{5} \mathrm{http}: / /$ www.aradueycampos.org/ficheros/MANUAL_TRINO_BUENAS\%20PR\%C1CTIC AS\%20AMPIENTALES.pdf
} 
- Potenciar en los territorios rurales la formación y la educación para la conservación ambiental.

- Dinamizar a los colectivos sobre las posibilidades de creación de empleo

- Mantener la biodiversidad con el fin de asegurar tanto la conservación de las especies como su inclusión en la oferta turística.

- Fomentar el uso de los recursos naturales salvaguardando la sostenibilidad y el crecimiento económico y social de los territorios rurales

- Apoyar la configuración de una oferta regional de turismo ornitológico.

\section{Proyecto RETO ${ }^{6}$ (Red de Espacios de Turismo Ornitológico)}

Nace como consecuencia de la experiencia previa en el LEADER II del Proyecto transnacional GRUS PROYECT, en el cual participaron diversos grupos españoles en colaboración con otros del resto de Europa.

Una de las acciones del proyecto se centra en la "Creación y desarrollo del producto turismo ornitológico". En esta acción se establece como objetivo: "conocer con profundidad la demanda actual de turistas ornitólogos especializados y de turistas que observan aves como actividad complementarias".

El proyecto está integrado por Grupos de Acción Local españoles, de siete comunidades autónomas, y dos regiones francesas que cuentan con zonas de humedales y recursos ornitológicos.

Una de las acciones de este proyecto será la creación de paquetes turísticos, mientras que un porcentaje del presupuesto está orientado a promover acciones individuales de cada una de las regiones, como la formación de guías, la realización de talleres e intercambio con otros lugares de turismo ornitológico.

El objetivo principal de RETO es desarrollar una red de turismo ornitológico que se basa en tres pilares fundamentales: medio ambiente, turismo y nuevas tecnologías que implique al máximo número de agentes, tanto públicos como privados, y atraiga a un turismo rural de calidad, aprovechando los recursos naturales y culturales existentes.

${ }^{6}$ http://www.magrama.gob.es/es/ministerio/servicios/publicaciones/art_datos_art.asp?articul oid $=279 \&$ codrevista $=$ LEAD 


\section{Proyecto IBERAVES}

Se trata de un proyecto a nivel internacional, para desarrollar un curso de formación en turismo ornitológico en las áreas de la Red Natura 2000 de la zona europea. Se trata de formar en cuanto a la gestión y conservación de estos espacios, sobre la demanda, necesidades y recursos naturales protegidos. Este proyecto va orientado a los agentes que intervienen en la gestión y ejecución de la oferta turística, es decir, administración, empresarios y estudiantes.

\section{TURISMO ORNITOLÓGICO EN ARAGÓN}

La Comunidad Autónoma de Aragón, está bajo la protección de las mismas figuras con las que cuenta cualquier área que esté adherida a la Unión Europea. Las principales protecciones del territorio son las ZEPA y los LIC. Junto a estas actúa la Red Natural de Aragón, en la cual se integran tanto Parques Nacionales, Parques Naturales, Reservas Naturales, Monumentos Naturales y Paisajes Protegidos, los humedales de importancia internacional incluidos en el Convenio RAMSAR así como Reservas de la Biosfera.

\section{Proyecto LIFE- Naturaleza ${ }^{7}$}

Su objetivo principal, es ayudar a los países miembros a conservar la naturaleza para poder mantener, así como mejorar, los hábitats naturales, la flora y la fauna de los espacios que se encuentran dentro de la Red Natura 2000.

Los principales proyectos de esta rama en Aragón son:

- La Conservación del hábitat del Cernícalo primilla: Este Plan se aprobó en el 2000, el cual estaba orientado al mantenimiento y mejora de su hábitat y sobre todo en aquellos lugares en los que anidan.

- Adecuación de tendidos eléctricos en las ZEPA de Aragón: Con esta actuación se pretendía reducir las colisiones en los tendidos eléctricos de las aves y más concretamente aquellas que se encuentran en peligro de extinción.

- Conservación de Margaritifera auricularia en Aragón: Se pusieron en marcha unas directrices para el mantenimiento y mejora de sus hábitats en especial en aquellas zonas encontradas dentro de los LIC.

\footnotetext{
${ }^{7}$ http://www.aragon.es/DepartamentosOrganismosPublicos/Departamentos/AgriculturaGan aderiaMedioAmbiente/AreasTematicas/MA_Biodiversidad/ProyectosEuropeos?channelSel ected $=4 \mathrm{ab} 736552883 \mathrm{a} 210 \mathrm{VgnVCM} 100000450 \mathrm{a} 15 \mathrm{acRCRD}$
} 
- Proyecto LIFE Antídoto: Éste pretendía crear una nueva estrategia contra el envenenamiento de animales carnívoros y posteriormente de las aves necrófagas que se alimentaban de dicho animal envenenado.

\section{Programa VULTOURIS}

La red de Turismo Ornitológico VULTOURIS ${ }^{8}$, se desarrolla desde el año 2009 y está formada por seis centros de interpretación de la Naturaleza de la Comarca de la Hoya de Huesca, Navarra y el valle d'Ossau (Francia). Esta Red Transpirenaica tiene como principal interés el trabajo sobretodo con tres tipos de aves emblemáticas de estos territorios: el buitre leonado, el quebrantahuesos y el alimoche.

El proyecto Vultouris tiene una serie de objetivos:

- Establecer una Red para el fomento del Turismo Ornitológico a través de entidades y de sus Centros de Interpretación de la Naturaleza dedicados a las aves

- Proteger y divulgar el patrimonio natural de las aves carroñeras pirenaicas

- Reforzar la identidad transfronteriza de la población en sus territorios

El proyecto finalizó el 31 de marzo de 2012 habiendo desarrollado una gran variedad de acciones con el objetivo de poner en valor el territorio pirenaico a través de sus recursos ambientales, y promoviendo el conocimiento y la conservación de 3 especies emblemáticas.

\section{Rutas Ornitológicas}

Éstas se encuentran principalmente en zonas de protección ambiental o faunística, por lo que el turista se encuentra en pleno contacto con la naturaleza y pueden disfrutar del hábitat de la avifauna que se da en esa área.

- Laguna de Gallocanta

- Lomaza de Belchite

- Espacio Natural Protegido Sierra de Guara

- Espacio Natural Protegido Posets-Maladeta

- Espacio Natural Protegido Valles Occidentales

- Paisaje Protegido de los Pinares de Rodeno

- Parque Natural del Moncayo

\footnotetext{
${ }^{8}$ http://www.vultouris.net/index.php
} 


\section{Asociaciones}

Existen diversas asociaciones cuyo fin común es la protección y conservación de las aves y sus hábitats, las cuales realizan diversas actividades y proyectos para lograr su objetivo.

\section{Fundación para la Conservación del Quebrantahuesos (F.C.Q)}

Esta fundación ${ }^{9}$ se creó en 1995, es una organización no gubernamental, que se dedica a promover y desarrollar proyectos de conservación, gestión, investigación, desarrollo rural y sensibilización en los hábitats de montaña en los que vive el quebrantahuesos.

\section{Fondo Amigos del Buitre (F.A.B)}

Esta asociación ${ }^{10}$ es una entidad conservacionista sin ánimo de lucro, fundada en 1986, tiene como fin la protección, conservación y divulgación de las aves carroñeras y sus hábitats.

Sus principales actuaciones son la manutención de comederos artificiales para buitres, alimoches y milanos, así como cebaderos para el quebrantahuesos en el Pirineo aragonés y áreas circundantes.

Uno de sus proyectos consiste en la creación y posterior manutención de comederos, que pretenden sustituir a los antiguos muladares en áreas controladas sanitariamente, donde la diversificación de alimento se evalúa y orienta a los niveles óptimos de aprovechamiento por parte de las aves.

\section{Grupo Ornitológico Oscense (G.O.O)}

Este grupo ornitológico ${ }^{11}$ se dedica a la observación, el estudio y la defensa de las aves silvestres. Es la ONG naturalista más antigua de la provincia de Huesca (data de 1980). Las primeras jornadas ornitológicas se dieron en 1987.

El grupo ornitológico realiza diversas actividades cono: anillamiento de aves, excursiones, cursillos y trabajan para la conservación de las aves.

\footnotetext{
${ }^{9} \mathrm{http} / /$ www.quebrantahuesos.org/htm/es/inicio/control?zone=pub\&sec=ppal\&pag=inicio\&loc=es

${ }^{10} \mathrm{http}: / / \mathrm{www}$. fondoamigosdelbuitre.org/

${ }^{11} \mathrm{http}: / / \mathrm{www}$. avesdehuesca.es/
} 


\section{TURISMO ORNITOLÓGICO EN LAS COMARCAS DE ESTUDIO (HOYA DE HUESCA Y SOMONTANO DE BARBASTRO)}

Se ha realizado un estudio del ámbito territorial de ambas comarcas así como las actuaciones de fomento llevadas a cabo en cada una de ellas. Igualmente se ha investigado sobre los centros de interpretación y los observatorios de aves que en ellas existen y las rutas ornitológicas que comprenden cada área.

\section{Comarca de la Hoya de Huesca}

La Comarca Hoya de Huesca es una de las diez comarcas que constituyen la provincia de Huesca. Situada entre los ríos Sotón y Alcandre y limitada por las sierras de Gratal y Guara por el norte y la comarca de Los Monegros por el sur. Su territorio tiene una extensión de $2.525,60 \mathrm{~km}^{2}$ y está formada por 40 municipios que comprenden más de 120 núcleos de población con un total de 68.186 habitantes.

El área de estudio es el noroeste de la provincia, abarcando 17 de sus municipios: Agüero, Alerre, Arguis, Ayerbe, Banastás, Biscarrués, Casbas de Huesca, Chimillas, Igriés, La Sotonera, Loarre, Loporzano, Loscorrales, Murillo de Gállego, Nueno, Peñas de Riglos (Las) y Santa Eulalia de Gállego. Con un total de población de 7.054 habitantes.

Además del estudio del Prepirineo, he decidido incluir una zona del llano de la comarca de la Hoya de Huesca, más concretamente, la zona de Lupiñen-Ortilla y La Sotonera, ya que en los meses de febrero y marzo es una zona de gran importancia en cuanto al avistamiento y estudio de aves migratorias, especialmente de la Grulla Común, ya que aprovechan este punto para descansar y así reponerse antes de cruzar los Pirineos en su largo viaje migratorio.

\section{Actuaciones de fomento: Plan de Dinamización}

El Plan de Dinamización de Producto Turístico de la Comarca Hoya de Huesca comienza en Septiembre de 2006 tras la firma del Convenio por varias de las delegaciones competentes en la materia, como el Ministerio Comercio, Industria y Turismo, la Comunidad Autónoma de Aragón, la Comarca de la Hoya de Huesca, la Asociación de Empresarios de hostelería de la provincia de Huesca y la Asociación Comarcal del Empresarios turísticos, con el objetivo del aprovechamiento de las potencialidades económicas y turísticas que la comarca de la Hoya ofrece tanto en productos de naturaleza, cultura, deporte, etc.

Lo que se pretende es favorecer el desarrollo turístico de la comarca de La Hoya de Huesca, ya que por sí misma tiene elementos característicos para la atracción de la demanda turística. 
Mapa $\mathbf{n}^{\mathbf{0} 1}$. Delimitación del territorio a estudio

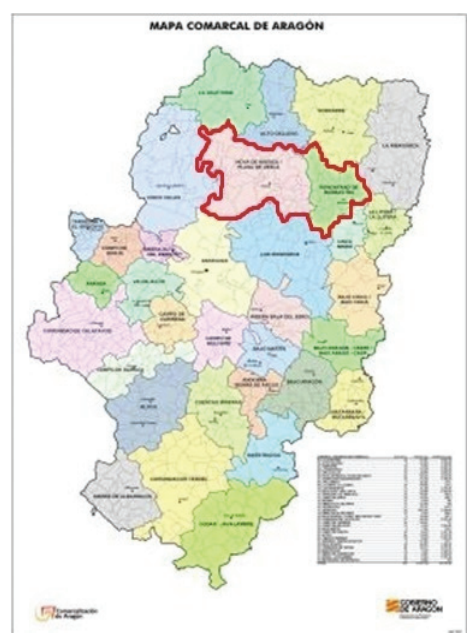

Fuente: www.comarcas.es

En este Plan de Dinamización, dentro del apartado de naturaleza, existe un subapartado de turismo ornitológico en el que se pretende conocer las potencialidades y los recursos de la zona, saber la demanda de este tipo de turismo, estudiar la competencia y estructurar una oferta para satisfacer a las necesidades de los visitantes. Igualmente dentro de éste se acondiciona los accesos al centro de interpretación de aves ARCAZ en Riglos, así como la mejora del edificio y el entorno y las infraestructuras y equipamientos que en él existen y se señalan diversas rutas de interés ornitológico.

\section{Centros de información y avistamiento de aves}

Estos centros están repartidos por todo el territorio, informando sobre las características geomorfológicas, climatológicas, etc. y las aves que nos podemos encontrar en cada tipo de entorno como por ejemplo, rupícolas, acuáticas o esteparias.

- Centro de aves Arcaz

- Mirador de los Buitres en Sarsamarcuello

- Santa Cilia de Panzano

- Alberca de Alboré 


\section{Rutas ornitológicas}

- Ruta ornitológica de la Sotonera

- Subida al Pico del Águila

- Ruta ornitológica de la Serreta

- Embalse de la Peña-Central de Carcavilla

- Embalse de las Navas

- Zona del pantano de la Peña

- Los Mallos de Riglos

- Recorrido por la Alberca de Loreto

\section{Comarca del Somontano de Barbastro}

La comarca del Somontano de Barbastro es una de las diez comarcas que constituyen la provincia de Huesca. Territorio de transición entre las sierras exteriores de los Pirineos y las llanuras del valle del Ebro. Su territorio tiene una extensión de 1.166,60 $\mathrm{km}^{2}$ compuesto por 29 municipios que comprenden alrededor de 58 núcleos de población con un total de 24.428 habitantes.

Los municipios de estudio son los comprendidos en la zona del Prepirineo. Abarca un total de 13 núcleos: Abiego, Adahuesca, Alquézar, Bierge, Colungo, El Grado, Estada, Estadilla, Hoz y Costeán, Naval, Olvena, Salas altas y Santa María de Dulcis. Con un total de población de 3.742 habitantes.

\section{Actuaciones de fomento: Plan de Dinamización}

Este plan es un convenio entre el Ministerio de Economía, Gobierno de Aragón, Comarca del Somontano de Barbastro y las Asociaciones de Empresarios de Barbastro y la Sierra de Guara. El objetivo principal de este Plan es aumentar el crecimiento del destino al mismo tiempo que se asegura su sostenibilidad. Las acciones llevadas a cabo son sobre todo de puesta en valor de los recursos turísticos, mejoras en el medio urbano, creación de nuevos productos, diversificación de la oferta turística, incremento de la calidad y sensibilización de la población.

Más en concreto sobre ornitología, se llevo a cabo el "Plan Turístico Ornitológico de la Comarca del Somontano de Barbastro", pero todavía no está implantado por falta de financiación. En primer lugar se ejecutó un diagnóstico territorial de toda la comarca, para luego centrarse en las tres áreas ornitológicas: la zona rupícola, de ribera y la cerealista o esteparia. Identificaron además las especies de cada zona. Seguidamente hicieron un breve estudio sobre la demanda, haciendo hincapié en las especies que suelen observar, con la siguiente implantación de 14 posibles rutas ornitológicas en el territorio. 
Gráfico n¹. Procedencia por CC.AA. de los turistas españoles

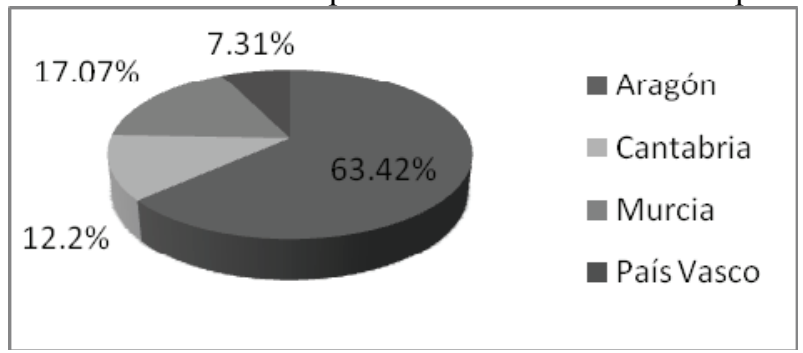

Fuente: Elaboración propia a partir de los datos obtenidos en la encuesta

Gráfico n⿳20: Procedencia por países de los turistas extranjeros

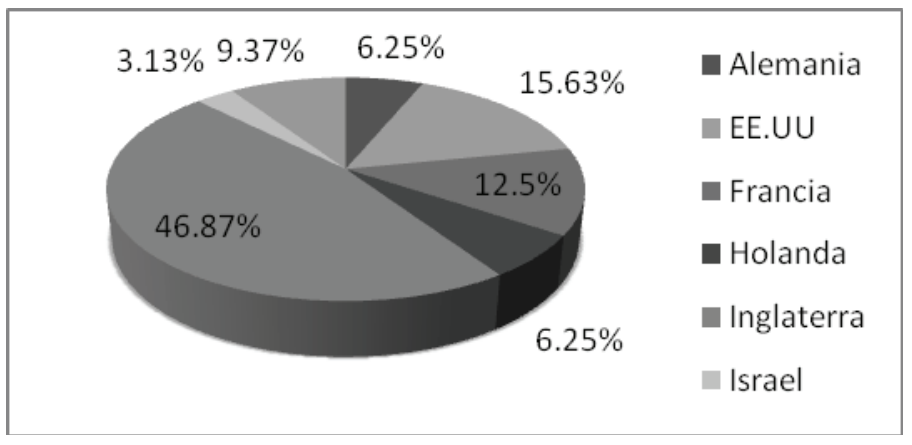

Fuente: Elaboración propia a partir de los datos obtenidos en la encuesta

\section{Centros de información y avistamiento de aves}

Centro del Parque Cultural del Río Vero

- Centro de interpretación del Espacio Natural Protegido de la Sierra y Cañones de Guara de Bierge

\section{Rutas ornitológicas}

Ruta de las Pasarelas del Vero

- Ruta de Chimiachas

- Ruta de las Fuentes del Puntillo y la Tamara

- Ruta Real de Mascún (también señalizada como sendero Losa Mora-Otín)

- Ruta del Huevo de Morrano

- Senda de los Carrizos y observatorio de aves El Grado

- El Grado-Olvena 
Gráfico no 3 y 4: Estudio de los turistas españoles y extranjeros en cuanto al género

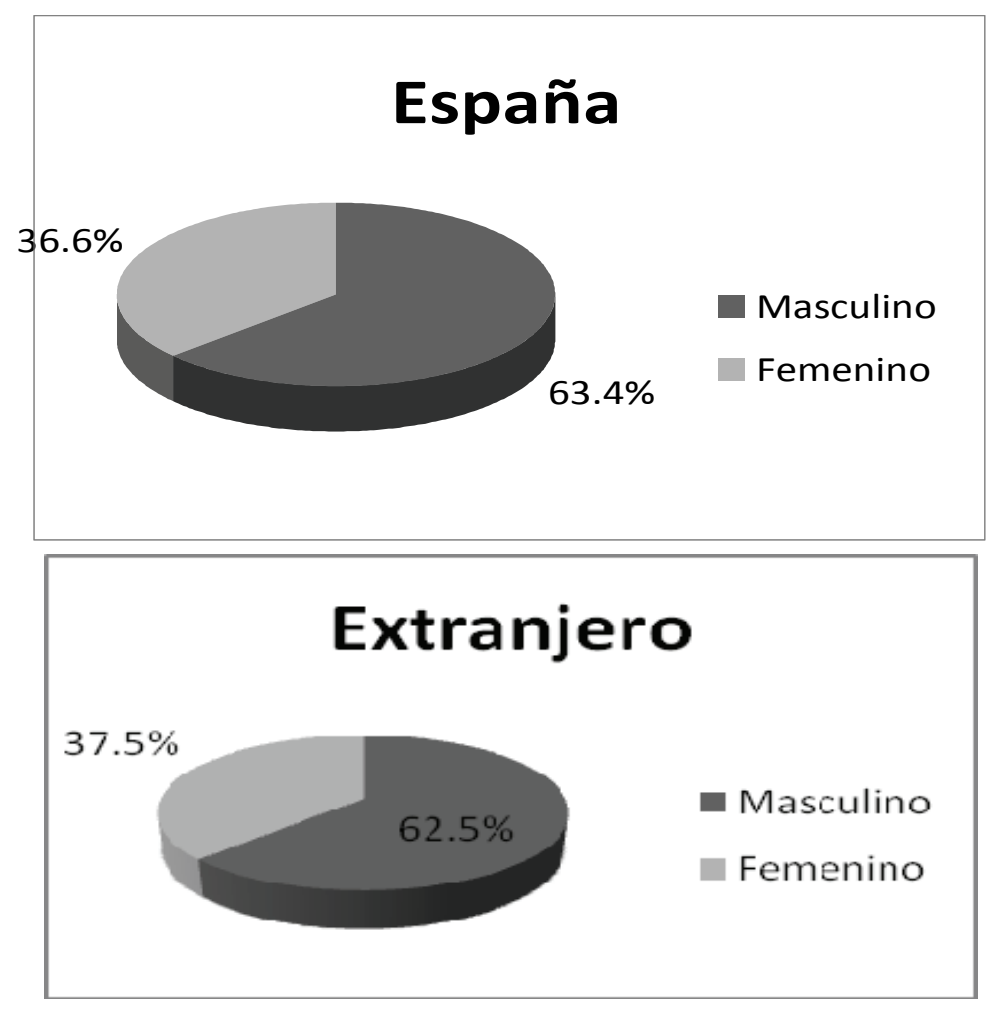

Fuente: Elaboración propia a partir de los datos obtenidos en la encuesta 
Gráfico n5 y 6: Turistas españoles y extranjeros en cuanto al rango de edad

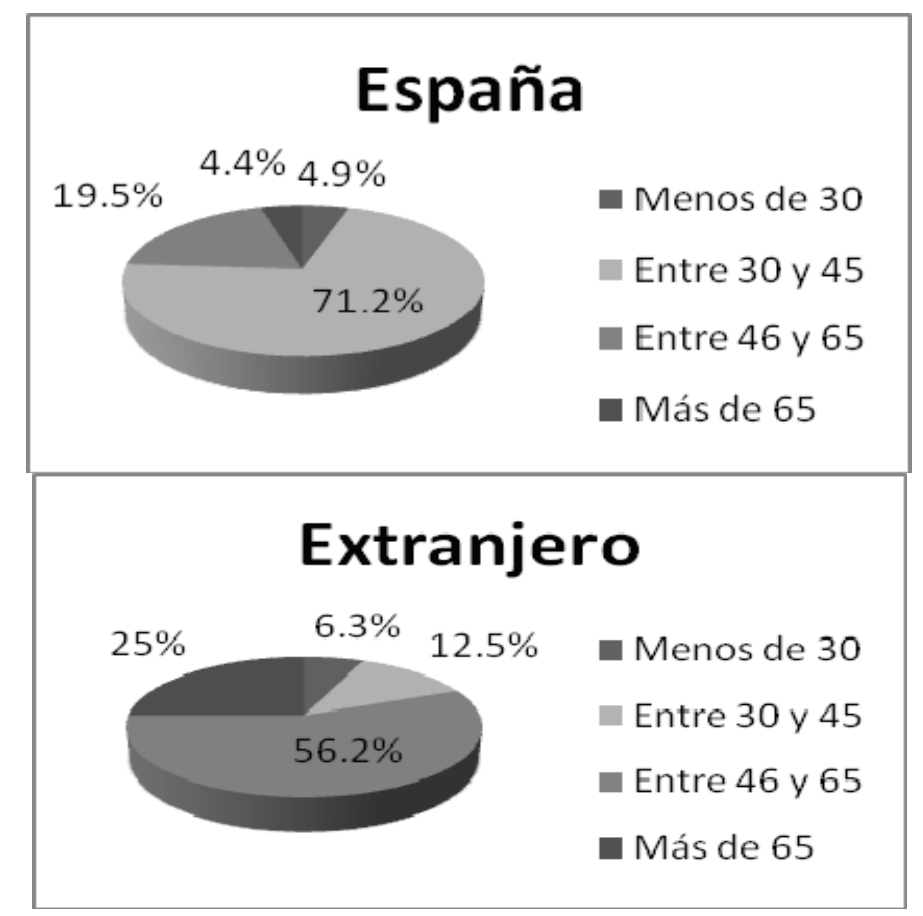

Fuente: Elaboración propia a partir de los datos obtenidos en la encuesta 
Gráfico n6 y 7: Turistas españoles y extranjeros en cuanto a su situación laboral

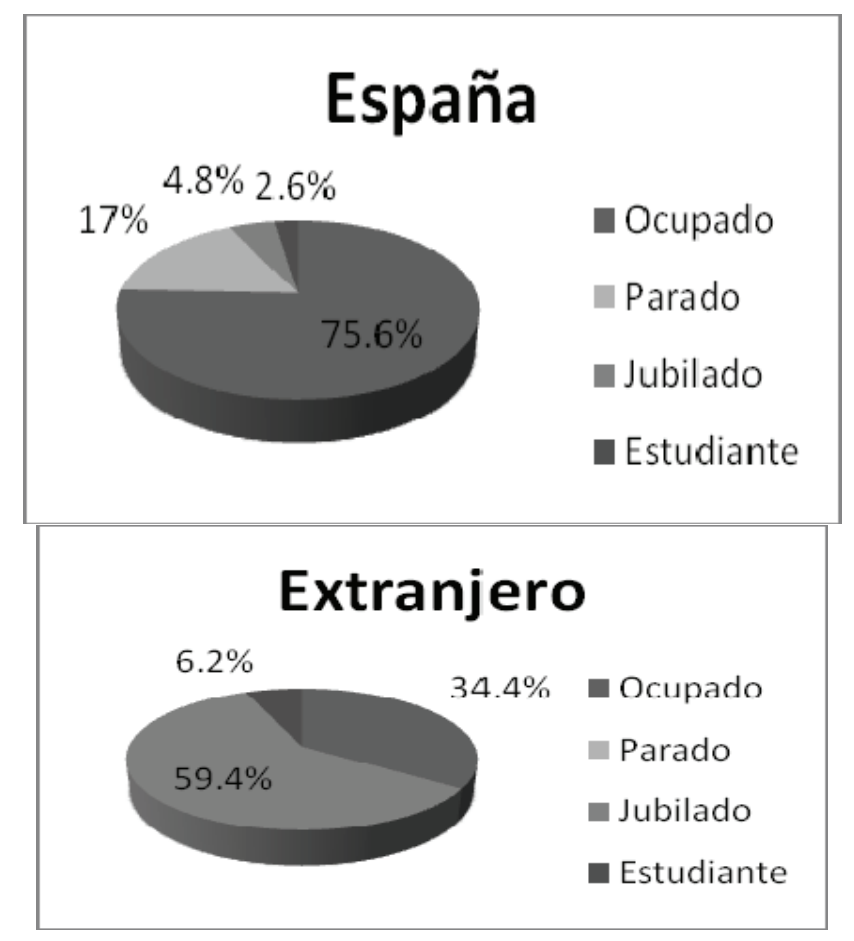

Fuente: Elaboración propia a partir de los datos obtenidos en la encuesta 
Gráfico $\mathbf{n}^{\mathbf{0} 7}$ y 8: Turistas españoles y extranjeros en cuanto a su nivel de estudios

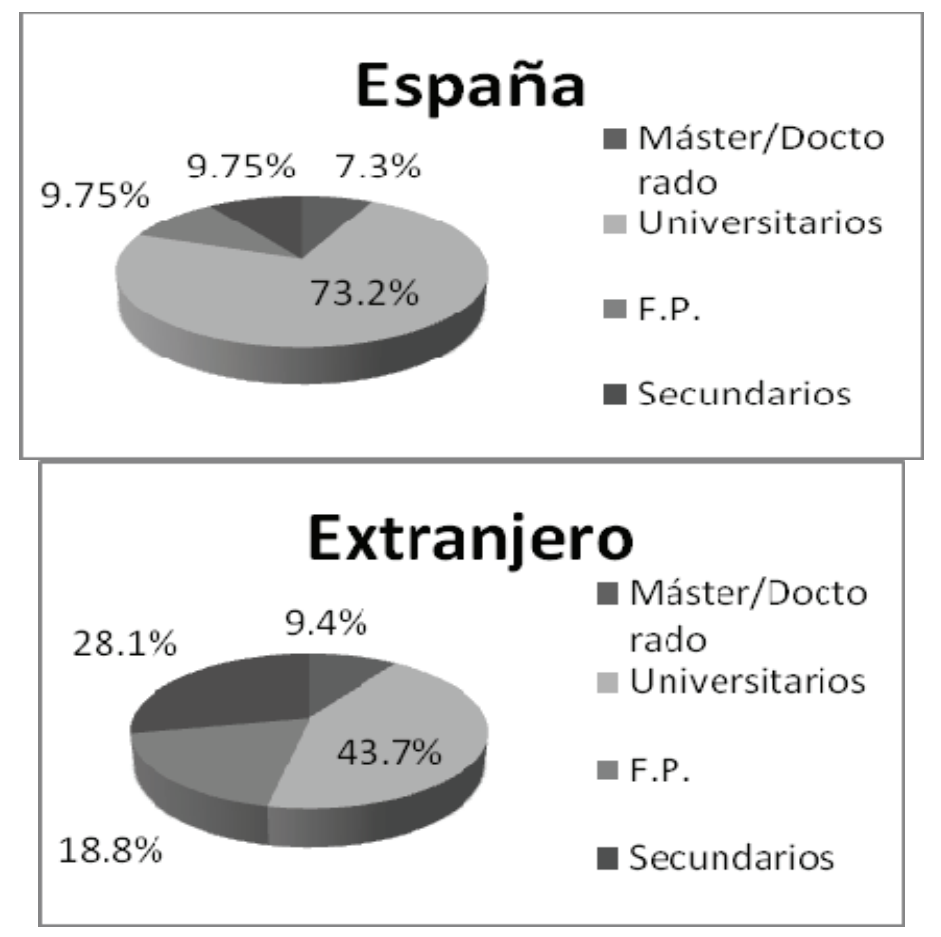

Fuente: Elaboración propia a partir de los datos obtenidos en la encuesta 
Gráfico n⿳9 y 10: Turistas españoles y extranjeros en cuanto a su grado de experiencia

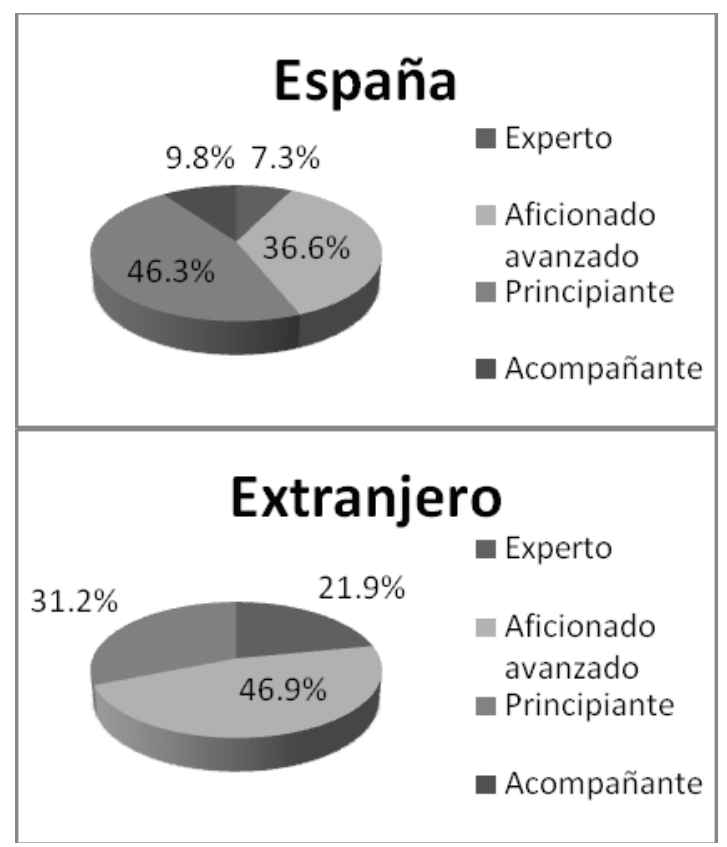

Fuente: Elaboración propia a partir de los datos obtenidos en la encuesta

\section{ANÁLISIS Y RESULTADOS}

De las encuestas realizadas se desprenden los siguientes resultados:

Más de la mitad de los encuestados $(56,2 \%)$ son españoles, mientras que los visitantes extranjeros suponen el porcentaje restante, es decir un 43,8\%. Es destacable por lo tanto el interés de éstos últimos por el avistamiento de aves.

Al estudiar la procedencia de los turistas ornitológicos por Comunidades Autónomas, la región predominante es Aragón, con un 63,42\%, seguida de Murcia con un $17,07 \%$, en tercera posición está Cantabria con un $12,2 \%$ y en último lugar el País Vasco con un 7,31\%.

El porcentaje más elevado de turistas extranjeros corresponde al Reino Unido, con un 46,57\%, seguido de EE.UU. con un $15,63 \%$. En tercer lugar, Francia $(12,5 \%)$ y Sudáfrica $(9,37 \%)$ son cuantitativamente importantes, mientras que Holanda y Alemania con un $6,25 \%$ y finalmente Israel $(3,13 \%)$ son menos representativos. Llama la atención el porcentaje de Estados Unidos y el de Sudáfrica por la distancia de los países emisores. 
Las personas que más viajan por motivos de avistamiento de aves son los hombres con un $63 \%$ frente al $37 \%$ que representan las mujeres interesadas en este tipo de turismo.

El rango de edad del turista español se encuentra principalmente entre 30 y 45 años con un $71,2 \%$ de los encuestados, le sigue con un $19,5 \%$ aquellos que están entre 46 y 65 . El $4,9 \%$ son menores de 30 años mientras que el 4,4\% son mayores de 65 años. En cuanto al turista extranjero la actividad se centra en personas de entre 46 y $65(56,2 \%)$ y un $25 \%$ tienen más de 65 años. El $12,5 \%$ de los encuestados tiene entre 30 y 45 años, mientras que el $6,3 \%$ no alcanzan los 30 años.

Observamos que en España predomina el visitante ocupado con un 75,6\%, seguido de un $17 \%$ de turistas parados. El 4,8\% corresponde a los jubilados, mientras que los estudiantes representan un 2,6\% del total. En cuanto a los turistas extranjeros un $59,4 \%$ son jubilados, seguidos de un $34,4 \%$ de turistas que están empleados y estudiantes con un $6,2 \%$ del total.

En cuanto al nivel de estudios el $73,2 \%$ del turista nacional tienen niveles universitarios, le siguen con un $9,75 \%$ los turistas con estudios de F.P. y secundarios, siendo los estudios de máster y doctorado el 7,3\%. Si nos centramos en el turista extranjero, el $43,7 \%$ poseen estudios universitarios, seguidamente y en orden descendente de porcentaje están los que tienen estudios secundarios $(28,1 \%)$, F.P $(18,8 \%)$ y algún máster o doctorado $(9,4 \%)$.

En cuanto a la frecuentación de visita, por un lado tenemos al turista español, del cual obtenemos que el $39 \%$ ha visitado el territorio por primera vez, un $34 \%$ lo han visitado 2-3 veces, seguidamente han estado de 4 a 6 veces un $17 \%$, mientras que un $10 \%$ de los turistas han estado en la zona más de 6 veces. Por otra parte el turista extranjero que visita la zona por primera vez es un $56 \%$, seguidos de aquellos que lo hacen 2-3 veces con un $25 \%$ y en último lugar están los que han estado más de 6 veces (19\%).

El medio de transporte varía en función de la procedencia del turista. Todos los visitantes españoles que se encuentran en el área de estudio, utilizan su vehículo propio para desplazarse, mientras que el $56 \%$ de los turistas extranjeros que nos visitan, utilizan un vehículo de alquiler.

Se pudo observar que un $64,4 \%$ de los visitantes prefieren realizar estos viajes acompañados por amigos, mientras que un $38,4 \%$ lo hacen en familia. En orden descendente se encuentran aquellos que viajan en pareja $(23,3 \%)$ seguidos por grupos organizados $(20,5 \%)$ y finalmente los que viajan solos $(9,6 \%)$.

Existe una gran disparidad de resultados entre turistas españoles y extranjeros en cuanto a la duración de la estancia en el territorio. Mientras que en el turista español predomina en los viajes sin pernocta $(53,6 \%)$ y las estancias de 3-4 noches con un $22 \%$ del total, seguidos de estancias de 5-6 noches $(17,1 \%)$ y en último lugar las estancias de 1 ó 2 noches que suponen el 7,3\%. El turista extranjero prefiere realizar unas estancias más largas, siendo la opción de más de 6 noches la 
más recogida (43,8\%), en segunda opción están la pernoctación de 3-4 noches con un $31,2 \%$. Con el mismo porcentaje un $12,5 \%$ se encuentran los viajes de una duración de 1 ó 2 noches y los de 5-6 noches.

A la hora de indicar el establecimiento, la opción mayoritaria marcada por el turista español, fue con un 47,5\% la opción de "otros" el cual es el hostal, furgoneta camper y casa de amigos. Con un $42 \%$ la segunda opción es el camping y la casa rural en último lugar con un $10,5 \%$.

En cuanto al turista extranjero el hotel rural supone un 53,1\%, seguidamente de la casa rural con un $25 \%$. En tercer lugar se encuentra el hotel (15,6\%), para finalizar con "otros alojamientos" $(6,3 \%)$ que en este caso es segunda residencia.

Se observa que la mayoría de los turistas ornitológicos $(75,3 \%)$ realiza este tipo de turismo por hobby, seguidamente por relax con un 39,7\% y por ser una nueva experiencia $(32,9 \%)$, en último lugar están aquellos que lo realizan por trabajo con un $15,1 \%$.

Además de realizar turismo ornitológico, un $90,4 \%$ de los encuestados lo complementa con actividades en el medio natural, seguido por un $79,5 \%$ por actividades culturales. En tercer lugar se encuentra la gastronomía con un $72,6 \%$, con un porcentaje menor, un $38,4 \%$ se encuentra el ocio, seguidamente el enoturismo con un $28,8 \%$ y las actividades acuáticas (17,8\%). Las actividades menos realizadas por los turistas están el golf $(2,7 \%)$ y las actividades aéreas con un $1,4 \%$.

El grado de interés ha sido valorado del 1 (mínimo) al 5 (máximo) en las encuestas. La actividad mejor valorada es la que se realiza en el medio natural, que alcanza una media de 4.19. Las actividades culturales tienen un 4.14, mientras que la gastronomía tiene una media de 3.88. En cuarto lugar se encuentra el ocio cuya valoración es 2.95 de media. El enoturismo consigue una valoración indiferente con un 2.29 y ya en decrecimiento encontramos las actividades acuáticas (2.14), aéreas (1.16) y el golf (1.11).

A la hora de captar la información del destino, el $67,1 \%$ de los turistas han recogido información gracias a conocidos. La opción de Internet es la segunda más señalada con un $34,2 \%$. Un $19,2 \%$ de los visitantes se informan a través de los folletos. Las revistas especializadas en ornitología son otra fuente a la que recurren un $16,4 \%$ y las asociaciones ornitológicas suponen un $13,7 \%$. En contraposición, las menos consultadas son los documentales $(4,1 \%)$ y ninguno de ellos ha consultado información ornitológica del territorio en ferias especializadas.

El aspecto mejor valorado por los turistas es la calidad del paisaje $(79,5 \%)$, seguido de la actividad ornitológica $(52,1 \%)$ y el alojamiento (47\%). En contraposición lo peor valorado son las infraestructuras de la zona con un 12,3\%, la señalización $(9,6 \%)$ y la actividad ornitológica con un $2,7 \%$ de los encuestados.

En el caso de España, en cuanto al grado de experiencia, podemos observar que el $46,3 \%$ de los encuestados son principiantes, el $36,6 \%$ son aficionados avanzados, en tercer lugar se encuentran los expertos con un $9,8 \%$, para finalizar 
con el 7,3\% que son acompañantes. En cuanto al turista extranjero el 46,9\% son aficionados avanzados, el $31,2 \%$ son principiantes, mientras que por último están los expertos con un $21,9 \%$.

El presupuesto del visitante varía en función de su procedencia. El 63,4\% del los turistas nacionales encuestados posee un presupuesto inferior a 50 euros, ya que son la mayoría visitantes que no pernoctan y únicamente tienen como gasto el transporte y en algunos casos la comida. Le sigue un $34,1 \%$ con un presupuesto entre 50 y 100 euros y finalmente aquellos que están entre 101 y 150 euros $(2,5 \%)$. El presupuesto del turista extranjero es más elevado, el $50 \%$ están entre 50 y 100 euros, mientras que un 40,6\% ha marcado la opción de entre 101-150 euros, en último lugar están aquellos cuyo presupuesto es menor a 50 euros $(9,4 \%)$.

Gráfico n¹1: Número de ocasiones que turistas nacionales e internacionales han visitado el destino

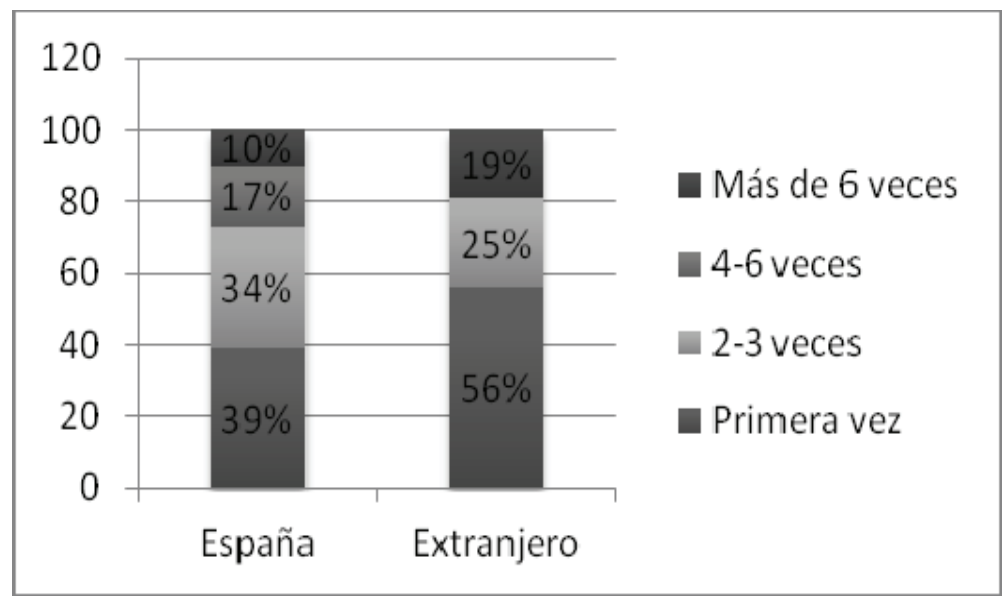

Fuente: Elaboración propia a partir de los datos obtenidos en la encuesta 
Gráfico n⿳12: Duración de la estancia según procedencia

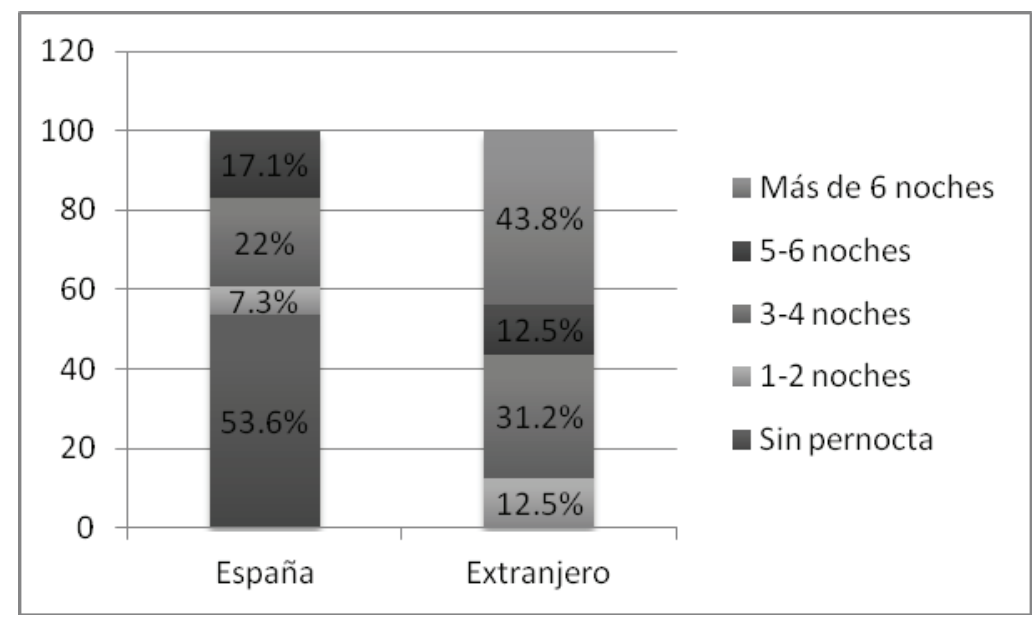

Fuente: Elaboración propia a partir de los datos obtenidos en la encuesta

Gráfico $\mathbf{n}^{\mathbf{0}}$ 13: Tipo de alojamiento según procedencia

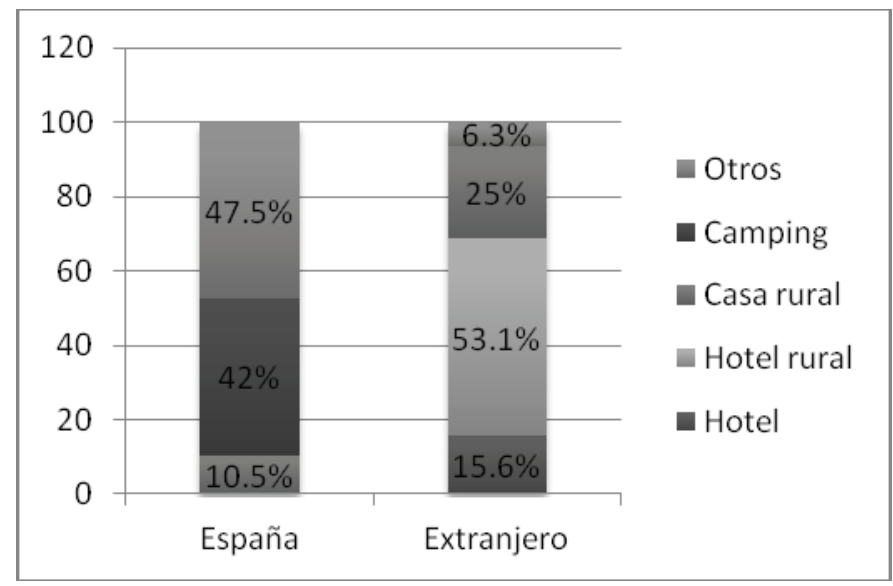

Fuente: Elaboración propia a partir de los datos obtenidos en la encuesta 
Gráfico n⿳14 y 15: Turistas nacionales y extranjeros en cuanto a su presupuesto por persona y por día

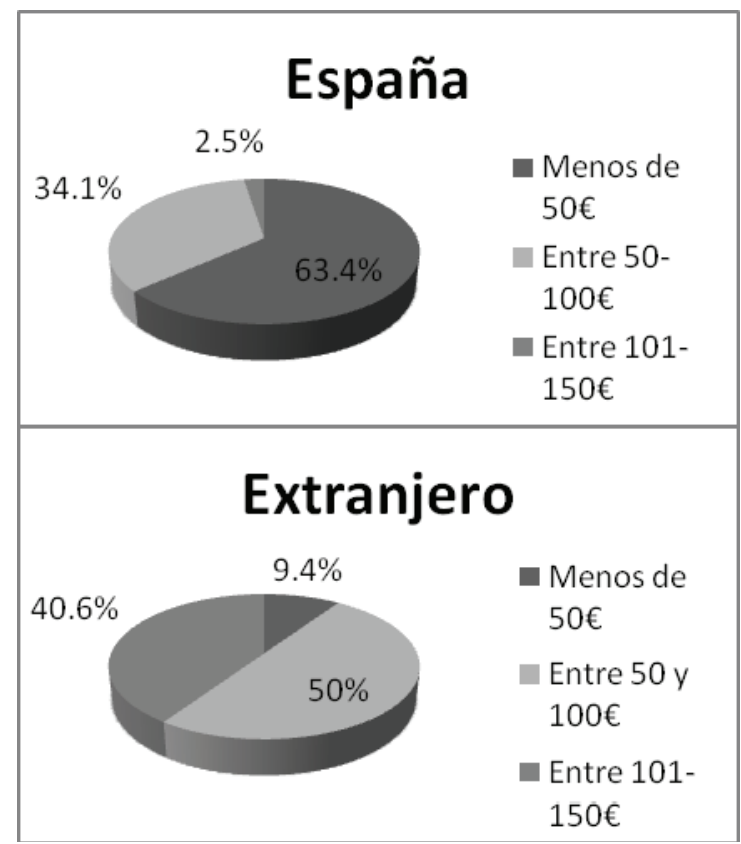

Fuente: Elaboración propia a partir de los datos obtenidos en la encuesta

\section{CONCLUSIONES E IMPLICACIONES}

No existe un turista ornitológico homogéneo en el territorio estudiado. Se podrían dividir en dos bloques:

Los turistas nacionales, los cuales se acaban de iniciar en la materia, la mayoría son principiantes, su rango de edad está entre 30 y 45 años, con un nivel de estudios elevado. Realizan sobre todo viajes en el día y si pernoctan lo hacen en hostales o campings. Suelen viajar con amigos o familiares, con un presupuesto inferior a 50 euros.

El turista extranjero, está en el territorio por más días, tienen un grado de experiencia en ornitología más elevado y su rango de edad se encuentra de los 45 en adelante, con un nivel de estudios inferiores al turista nacional. Suelen pernoctar en hoteles rurales o casas rurales, buscando el contacto con la naturaleza, teniendo muy en cuenta la calidad del paisaje así como la señalización y las infraestructuras. Igualmente viajan con familiares, amigos o grupos organizados. Tienen un presupuesto que se encuentra entre los 50 y 150 euros por persona y por día. 
Igualmente de las encuestas realizadas se desprenden una serie de conclusiones interesantes que apunto a continuación:

Las señales informativas de la zona, que ofrecen información adicional sobre el territorio al turista, se encuentran la mayoría en castellano, por lo que son ilegibles para los visitantes extranjeros, además de estar consideradas por éstos como de un fuerte impacto visual, ya que se encuentran poco integradas en el ecosistema. Se debería tener en cuenta la buena señalización y su correcto mantenimiento puesto que estos aspectos son los peores valorados por los visitantes. Otras señales informativas que se han ido divisando por la zona, al estar expuestas a las inclemencias del tiempo, estaban totalmente quemadas por el sol.

Los centros de interpretación ornitológicos orientados a dar información sobre la zona, fue uno de los aspectos peor valorados por los visitantes en la encuesta. La razón principal fue que éstos se encontraron con los centros cerrados y no podían disfrutar de las instalaciones. Sería aconsejable llevar un control de apertura de éstos, contemplando la flexibilidad horaria y que al menos los días festivos, se encontrasen abiertos al público.

Dado que el turista considera de gran importancia la calidad del paisaje y el entorno, incluso alguno de ellos lo ve fundamental para la realización del viaje, éste debe estar perfectamente conservado al igual que la biodiversidad que existe en él.

Se debería valorar la realización de una mayor promoción del turismo ornitológico del territorio por medio de ferias, folletos, revistas especializadas o documentales, ya que la gran mayoría de los turistas no se habían informado por estos canales de información, sino gracias a conocidos o Internet. En este caso sería la administración y las instituciones las encargadas de esta labor, de dar a conocer de una forma activa los recursos de la zona. Hay ferias importantes que se llevan a cabo tanto en España como en el extranjero, y son en éstas cuando el turista ornitológico se interesa por un destino en concreto. Habría que mejorar los medios de promoción y comercialización, así como disponer de una oferta conjunta y atractiva para al menos ser otro de los posibles destinos a la elección de la demanda.

Otro aspecto a destacar es que, con los turistas extranjeros que se pudo hablar más ampliamente, la valoración de la importancia de contratar a un guía local de la zona, ya que suelen ser mejores conocedores del territorio y de los principales puntos de avistamiento. Las instituciones están ayudando a que éstos se formen, pero a la hora de la contratación no se sabe dónde acudir para contactar con ellos. De allí que muchos de los grupos extranjeros que nos visitan, acudan al territorio con un guía desde su país de origen, para no tener ningún tipo de problema en el destino. 


\section{BIBLIOGRAFÍA}

BLANCO PORTILLO, R. (2012): "El turismo ornitológico como producto turístico". Revista de la red de expertos en turismo; primer semestre 2012, n ${ }^{\circ} 10$

DE FARIAS, G.B. Y ALVES, A.G.C. (2007): "Historical and conceptual aspects of ethno-ornithology". Vol. 20. No 1, pp. 91-100

DE JUAN ALONSO, JM. (2006): "El turismo ornitológico: concepto y mercados. Referencias al destino Extremadura". Estudios turísticos, $\mathrm{n}^{\circ}$ 169-170

FERNÁNDEZ TABALES, A, BELTRÁN DE CEBALLOS, CARMONA ARTEAGA, JM., MAYNAR AGUILAR, M. (2007): "El turismo ornitológico en España como modalidad emergente. Organización interna de la actividad y caracterización de la demanda". Papers de Turisme; $\mathrm{n}^{\mathrm{0}}$ 42, pp.41-56.

LÓPEZ ROIG, J. (2008): "Turismo ornitológico en el marco del postfordismo, una aproximación técnico-conceptual". Cuadernos de turismo, $\mathrm{n}^{\circ}$ 21; pp. 85-111

MOSS, S. (2004): "A bird in the bush a social history of birdwatching". London Aurum.

Proyecto Iberaves (2010): “Turismo ornitológico, respetuoso y sostenible. Un reto en Red Natura 2000"

Proyecto TRINO (2011): “Manual de buenas prácticas ambientales para empresas turísticas adheridas al Proyecto TRINO".

Revista Actualidad LEADER: "Red de espacios de turismo ornitológico. La ruta de las aves". pp.18-19.

Revista Actualidad LEADER: "Turismo ornitológico. A vista de pájaro”. MijadasTrujillo (Cáceres). pp.22-23

SEO/BirdLife. "Reservas ornitológicas de SEO/BirdLife en Aragón" (03/05/2012). 